\title{
Using GH-Method: Math-Physical Medicine to Investigate the Role of HbA1C in the Triangular Relationship with Weight and Blood Pressure
}

\author{
Gerald C. Hsu* \\ Medical Research Scientist, EclaireMD Fondation, 7 Oak Haven Way, Woodside, CA 94062 USA. \\ *Corresponding Author: Gerald C. Hsu, Medical Research Scientist, EclaireMD Fondation, 7 Oak \\ Haven Way, Woodside, CA 94062 USA.
}

\section{INTRODUCTION}

The author uses math-physical medicine approach to investigate three sets of correlation between:

(1) Weight vs. Glucose/HbA1C - daily average glucose, including both fasting plasma glucose (FPG) and postprandial plasma glucose (PPG).

(2) Weight vs. blood pressure (BP).

(3) Glucose/HbA1C vs. BP

\section{METHOD}

The selected time frame is from 4/1/2014 to $12 / 31 / 2018$ (1,735 days) with big data of $\sim 18,000$ metabolic conditions such as Weight, Glucose/A1C, and BP. This paper utilized both time-series and spatial analysis to investigate those triangular relationships.

\section{RESULTS}

The time-series analysis results show strong correlations among these three pairs of curves.

Weight vs. Glucose / A1C: 78\% / 78\%

Weight vs. BP: $62 \%$

Glucose / A1C vs. BP: 84\% / 85\% (1\% difference is due to numerical tolerance).
In spatial analysis, all of the three sets in the diagram demonstrate various degree of strong correlation between any two variables. In addition, the three diagrams have $94 \%$ to $99 \%$ of data covered by $+/-20 \%$ variance band. The reason for the lower slopes between weight vs. glucose is due to the fact that FPG has higher correlation with weight, whereas PPG does not. The most interesting observation is the exceedingly high correlation or higher slope existing between glucose vs. BP.

The major difference between influences from Glucose and $\mathrm{A} 1 \mathrm{C}$ is shown by the gap between two adjacent data clouds. This is due to the author's glucose and A1C data that suddenly dropped significantly duringtheperiod, the second half of 2014 through the first half of 2015, after following his developed mathematical metabolism model and some practical ways to control his diabetes. When he switched the $\mathrm{X}$-axis and $\mathrm{Y}$-axis in the spatial analysis graphic diagrams, the gap still exists in each diagram although the general orientations of data clouds are somewhat different.

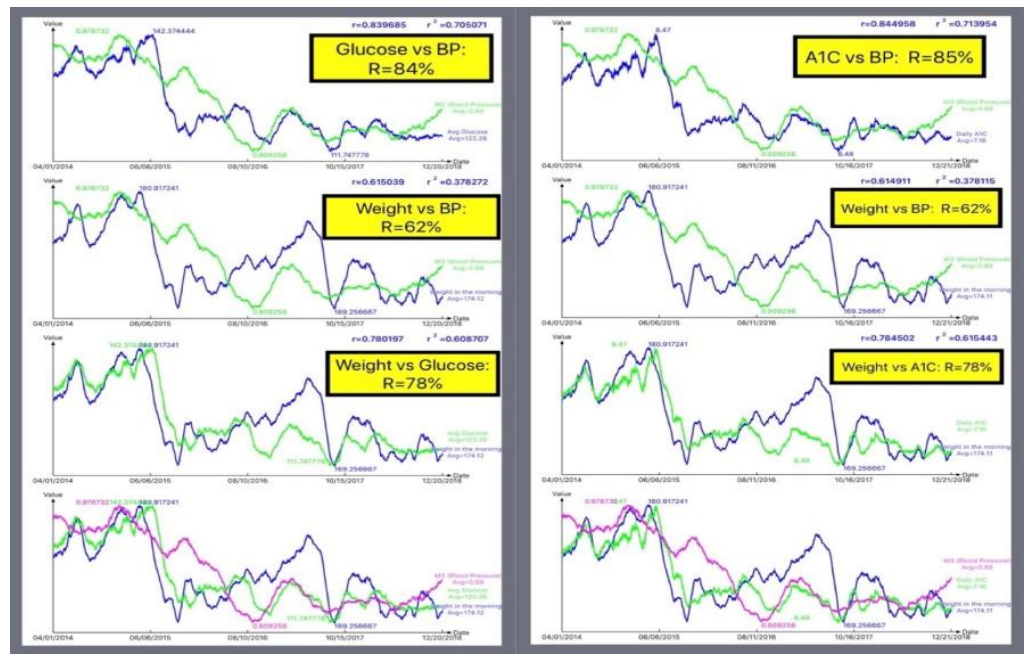

Figure 1: Time-series comparison between Glucose vs. Weight/BP and AlC vs. Weight/BP 
Using GH-Method: Math-Physical Medicine to Investigate the Role of HbA1C in the Triangular Relationship with Weight and Blood Pressure

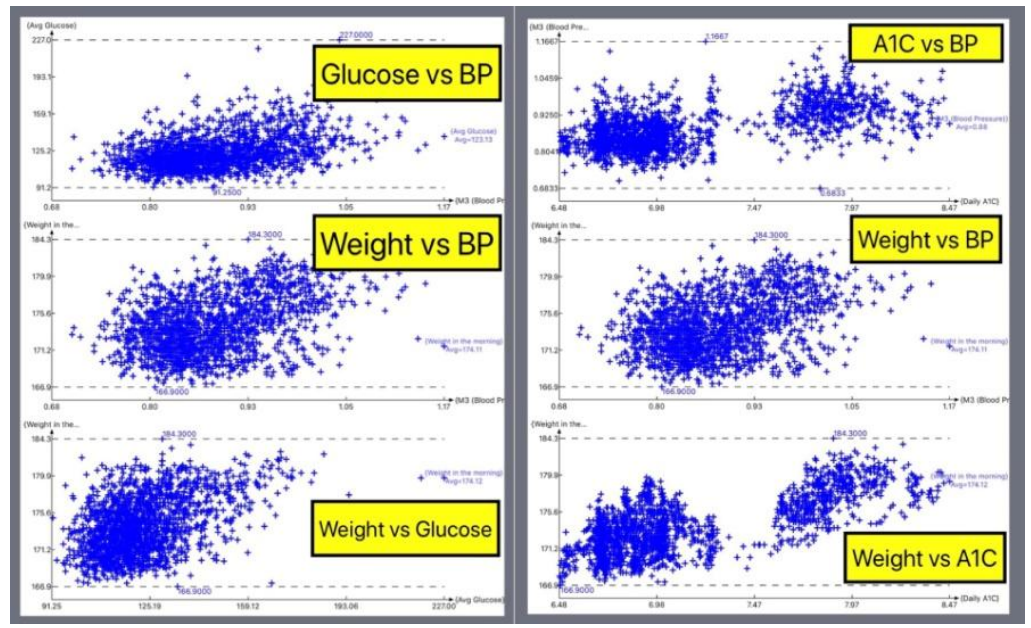

Figure 2: Spatial analysis comparison between Glucose vs. Weight/BP and AlC vs. Weight/BP

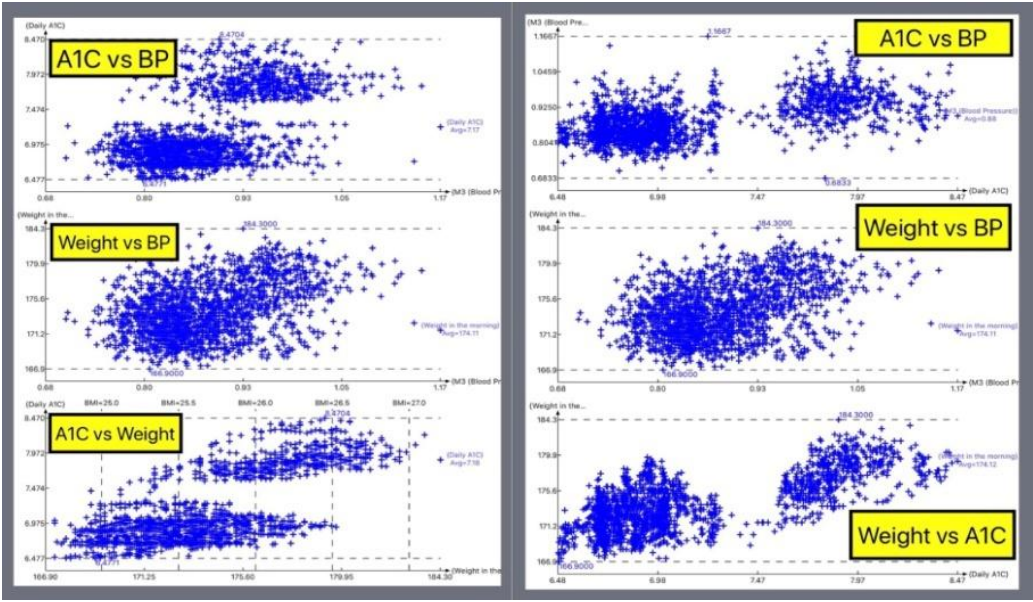

Figure 3: Observation of AlC variance via switching $X$-axis and $Y$-axis

\section{CONCLUSION}

By using the GH-Method: math-physical medicine approach, strong triangular relationships among weight, glucose/A1C and blood pressure are obvious. This confirms the many qualitative descriptions that existed in the medical community.

\section{REFERENCES}

[1] Hsu, Gerald C. (2018). Using Math-Physical Medicine to Control T2D via Metabolism Monitoring and Glucose Predictions. Journal of Endocrinology and Diabetes, 1(1), 1-6.

[2] Hsu, Gerald C. (2018). Using Signal Processing Techniques to Predict PPG for T2D. International Journal of Diabetes \& Metabolic Disorders, 3(2), 1-3.
[3] Hsu, Gerald C. (2018). Using Math-Physical Medicine and Artificial Intelligence Technology to Manage Lifestyle and Control Metabolic Conditions of T2D. International Journal of Diabetes \& Its Complications, 2(3), 1-7.

[4] Hsu, Gerald C. (2018, June). Using MathPhysical Medicine to Analyze Metabolism and Improve Health Conditions. Video presented at the meeting of the 3rd International Conference on Endocrinology and Metabolic Syndrome 2018, Amsterdam, Netherlands.

[5] Hsu, Gerald C. (2018). Using Math-Physical Medicine to Study the Risk Probability of having a Heart Attack or Stroke Based on Three Approaches, Medical Conditions, Lifestyle Management Details, and Metabolic Index. EC Cardiology, 5(12), 1-9.

Citation: Gerald C. Hsu, "Using GH-Method: Math-Physical Medicine to Investigate the Role of HbA1C in the Triangular Relationship with Weight and Blood Pressure”, International Journal of Research Studies in Medical and Health Sciences. 2020; 5(7): 06-07.

Copyright: (c) 2020 Gerald C. Hsu, This is an open-access article distributed under the terms of the Creative Commons Attribution License, which permits unrestricted use, distribution, and reproduction in any medium, provided the original author and source are credited. 